\title{
Ultrafiltration using graphene oxide surface-embedded polysulfone membranes
}

Taeseon Hwang ${ }^{\mathrm{a}}$, Joon-Suk Oh ${ }^{\mathrm{b}}$, Woosoon Yim ${ }^{\mathrm{a}}$, Jae-Do Nam ${ }^{\mathrm{c}}$, Chulsung Bae ${ }^{\mathrm{d}}$, Hyung-ick Kim ${ }^{\mathrm{e}}$ and Kwang Jin Kim ${ }^{* a}$

${ }^{a}$ Department of Mechanical Engineering, University of Nevada, Las Vegas, 4505 Maryland Parkway, Las Vegas, Nevada 89154, United States

${ }^{b}$ Center for Soft Matter Research, Department of Physics, New York University, 4 Washington Place, New York, New York 10003, United States

${ }^{c}$ Department of Polymer Science and Engineering, Sungkyunkwan University, 300 Chunchundong, Jangan-gu, Suwon, Gyeonggi-do 440-746, South Korea

${ }^{d}$ Department of Chemistry and Chemical Biology, Rensselaer Polytechnic Institute, 110 8th Street, Troy, NY 12180, United States

${ }^{e}$ Dongnam Regional Division / Manufacturing Process Technology Innovation Center' Korea Institute of Industrial Technology, 189 Dongjin-ro, Jinju-si, Gyeongsangnam-do, 660-805, South Korea

\section{SEPARATION AND PURIFICATION TECHNOLOGY}

* Corresponding author at: Department of Mechanical Engineering, University of Nevada-Las Vegas, 4505 Maryland Parkway Las Vegas, NV 89154-4027, USA. Phone: +1 702774 1419. E-mail address: kwang.kim@unlv.edu (K. Kim). 


\begin{abstract}
The effect of a hydrophilic layer of graphene oxide on polysulfone membranes was investigated to improve the antifouling properties of the membrane in ultrafiltration. Polysulfone membranes were obtained by the phase inversion method. Polyvinylpyrrolidone was added to generate pores in the membranes, and then a layer of graphene oxide was deposited on the prepared membranes using a vacuum-assisted filtration technique. This study investigated the cross-section/surface morphology, the water-contact angle, and the chemical structure of the prepared polysulfone/graphene oxide membranes. Due to the highly oxygenated structure of the graphene oxide, the surface hydrophilicity of the polysulfone membrane was significantly increased by applying a layer of graphene oxide. Moreover, the antifouling abilities of the membranes were evaluated by comparing the water-flux recovery ratio between the pure water flux before and after ultrafiltration.
\end{abstract}

Keywords: Ultrafiltration; Polymer membrane; Polysulfone; Graphene oxide; Vacuum-assisted filtering 


\section{Introduction}

During the last few decades, ultrafiltration technology has received increased attention due to its numerous advantages; for example, it has no phase changes, has low operating pressures, and employs ambient or relatively low operation temperatures [1]. Polysulfone (PSU) has been widely used as an ultrafiltration membrane due to its good thermal and chemical stabilities. However, the hydrophobic surface of PSU membranes induces severe fouling when exposed to protein-containing solutions [2]. This fouling reduces the flux of the membrane and increases operation costs by requiring an extra step in the cleaning process [3]. A major reason that proteins tend to adsorb onto the polymer membrane is due to a hydrophobic interaction between the surface of the membranes and the protein molecules [4,5]. Therefore, membrane fouling can be mitigated by modifying the hydrophobic membrane surface so that it can become more hydrophilic [6].

Based on this concept, many approaches have been tried to improve the hydrophilicity of PSU membranes. Polyethyleneoxide (PEO) and polyvinylpyrrolidone (PVP) have been added to increase hydrophilicity and improve the antifouling property of PSU ultrafiltration membranes $[7,8]$. In addition, efforts have been made to incorporate hydrophilic inorganic materials, such as titanium dioxide $\left(\mathrm{TiO}_{2}\right)$ [9], silica $\left(\mathrm{SiO}_{2}\right)$ [10,11], alumina $\left(\mathrm{Al}_{2} \mathrm{O}_{3}\right)$ [12], and zirconium dioxide $\left(\mathrm{ZrO}_{2}\right)$ [13] into the PSU matrix. Chemical modifications of PSU have been investigated as well, such as plasma treatment [14], sulfonation [15], and immobilization of hydrophilic segments onto polymers [16]. Unfortunately, only a marginal improvement in membrane antifouling properties and filtration efficiency has been accomplished due to minor enhancements in surface 
hydrophilicity [15]. Moreover, these methods have a limited capacity to increase the amount of nanoparticles and disperse them in the membrane casting solution.

In recent years, carbon nanomaterials, including carbon nanotubes and graphene, have been adopted as a new type of nanofiller to improve separation performance, mechanical strength, and thermal stability of polymer-based ultrafiltration membranes [17-19]. However, since these fillers inherently are hydrophobic, they cannot enhance the antifouling ability of the membranes. In contrast, graphene oxide (GO), an oxidized graphene, contains polar groups (hydroxyl and epoxide functional groups) on its basal planes, and carbonyl and carboxyl groups at the sheet edges; thus, GO easily can be dispersed into a polymer matrix because of its strong interactions of the polar functional groups with polymer chains [20]. Thus, when used as a nanofiller in polymer membranes, GO is expected to enhance the mechanical strength and thermal stability of the membrane and also improve the wetting properties of the hydrophobic polymer membrane due to the presence of its hydrophilic functional groups [21,22].

This study introduced a layer of GO onto PSU to improve hydrophilicity and antifouling abilities of the membrane. First, the PSU membrane was prepared by adding PVP, using a phase inversion method; then, a layer of GO was deposited on the membrane by means of a vacuumassisted filtration technique. The GO layer was expected to perform as a pre-filter of the prepared PSU membrane, which would prevent fouling on the membrane surface. Scanning electron microscopy (SEM) was employed to characterize the cross-section morphologies of the prepared membranes. Hydrophilicity of the membrane surfaces was characterized by measuring water contact angles. Furthermore, the antifouling ability of the prepared membrane was investigated 
by comparing the water flux recovery ratio (FRR) before and after ultrafiltration of the protein solution.

\section{Experimental}

\subsection{Materials}

PSU $\left(M_{W}=35,000\right.$, Fig. 1), PVP $(\mathrm{Mw}=360,000)$, and bovine serum albumin (BSA) were purchased from Sigma-Aldrich Corporation. Natural graphite flakes, sulfuric acid (95-97 \%, $\left.\mathrm{H}_{2} \mathrm{SO}_{4}\right)$, hydrogen chloride $(\mathrm{HCl})$, hydrogen peroxide $\left(30 \%, \mathrm{H}_{2} \mathrm{O}_{2}\right)$, potassium permanganate $\left(\mathrm{KMnO}_{4}\right)$, and $N$-methyl pyrollidone (NMP) were purchased from Sigma-Aldrich. Deionized (DI) water was used in the experiments. The chemical structures of polysulfone (a) and polyvinylpyrrolidone (b) are shown in Fig. 1.

(a)

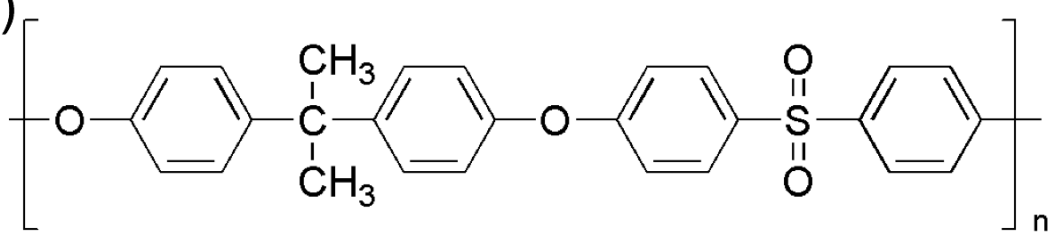

(b)

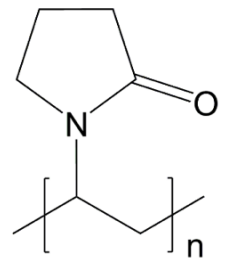

Fig. 1. Chemical structures of (a) polysulfone and (b) polyvinylpyrrolidone.

\subsection{Preparation of $G O$}

Natural graphite $(10 \mathrm{~g})$ was mixed with sulfuric acid $(250 \mathrm{~mL})$ in an ice bath, and then potassium permanganate ( $30 \mathrm{~g}$ ) was added to the mixture while stirring. The mixture was reacted for 2 hours at $35{ }^{\circ} \mathrm{C}$. Then, the reaction mixture was cooled to room temperature and diluted with of DI water $(1 \mathrm{~L})$. At that point, hydrogen peroxide $(30 \mathrm{~mL})$ was added to the mixture, and stirred for $20 \mathrm{~min}$ to remove impurities. The mixture was washed thoroughly with hydrogen chloride 
solution (5 \% in DI water) and DI water, several times. Subsequently, the mixture was collected and dried. GO dispersion was prepared by ultra-sonication resulting in a mixture of GO (50 mg) in DI water (50 mL) for 4 hours. The resultant dispersion was centrifuged (Combi-514R, Hanil Biomed Inc.) at 3,000 rpm for 15 min. After centrifugation, decantation was carried out by means of pipetting off the top $15 \mathrm{~mL}$ of the dispersion. Finally, the GO dispersion was prepared at a concentration of $0.1 \mathrm{mg} / \mathrm{mL}$.

\subsection{Preparation of PSU and PSU/GO membranes}

PSU membranes were prepared by the phase inversion method as shown in Fig. 2 [7,8]. PSU was the membrane matrix, and PVP was the membrane modifier as well as the pore-generating agent. PSU was dissolved in NMP and stirred at $60^{\circ} \mathrm{C}$ for 4 hours. At that point, various amounts of PVP were added and the PSU solution was stirred at $60{ }^{\circ} \mathrm{C}$ for an additional 4 hours. In order to eliminate bubbles in the solution, a vacuum was applied for $30 \mathrm{~min}$.

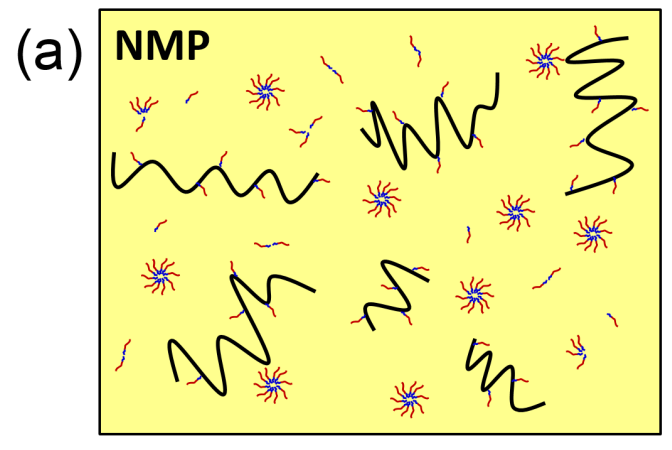

(b)

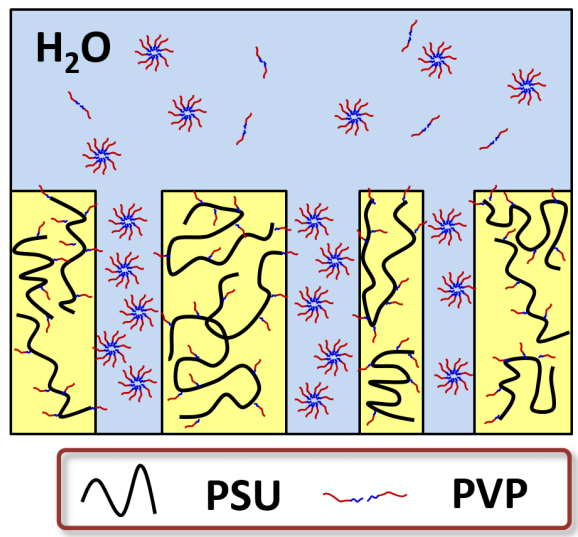

Fig. 2. The membrane formation process. (a) The PSU polymer and PVP were dispersed homogeneously in NMP and (b) the film was immersed in a DI water bath. This led to phase separation and the formation of ordered structure and pores in membrane by means of phase inversion. 
The solutions were cast on glass plates with a casting knife, and then immersed in a coagulation bath of DI water. In order to reduce error during phase inversion process, the volume and temperature of water bath were equalized. Subsequently, the pristine membranes were peeled off, and were washed thoroughly with DI water to remove residual solvent. These prepared membranes (a wet thickness of approximately $150 \mu \mathrm{m}$ ) were kept in DI water until used.

Fig. 3 shows that preparation of the PSU/GO membrane. This was carried out by vacuum filtration of the GO dispersion (2 mL) by using the as-prepared PSU membrane (10 wt. \% of PVP). The hydrophilic groups in the GO and membrane surface have been reacted through hydrogen boding reaction [23]. After this, extra GO was washed out with DI water to consolidate the layer of GO by eliminating weakly deposited GO. Details about types of samples and their compositions that were used in this experiment are summarized in Table 1.

(a)

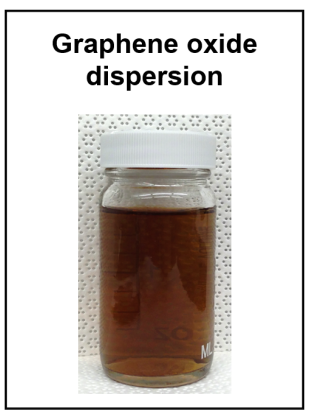

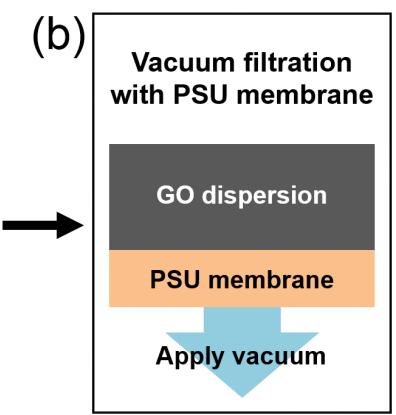

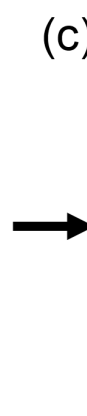

(c)

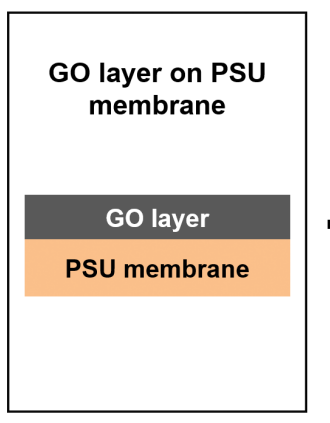

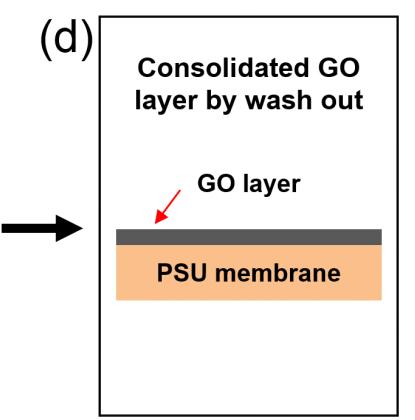

Fig. 3. Preparation of the PSU/GO membrane. First, (a) GO was dispersed in DI water. (b) Next, vacuum filtration of the GO dispersion took place throughout the PSU membrane and (c) the GO layer formed on the PSU membrane. Finally, (d) extra GO was washed out with DI water to consolidate the layer of GO. 


\section{Table 1}

Type of samples and their compositions of prepared ultrafiltration membranes.

\begin{tabular}{ccccc}
\hline Sample & PP0 & PP10 & PP20 & PSU/GO \\
\hline PVP content (\%) & 0 & 10 & 20 & 10 \\
PSU (g) & 5 & 4.5 & 4 & 4.5 \\
PVP (g) & 0 & 0.5 & 1 & 0.5 \\
NMP (g) & 20 & 20 & 20 & 20 \\
GO dispersion (mL) & - & - & - & 2 \\
\hline
\end{tabular}

\subsection{Characterizations}

Measurements from X-ray photoelectron spectroscopy (XPS) were performed using a monochromatic Al- $\mathrm{K} \alpha(1486.6 \mathrm{eV}) \mathrm{X}$-ray source at $13 \mathrm{kV}$ and $15 \mathrm{~mA}$ (ESCA 2000, VG Microtech). The thickness and cross-section morphology of the membranes both were investigated by using SEM (Mini-SEM, Evex Analytical Instruments, Inc. ) at $20 \mathrm{kV}$ and (JSM 7000F, JEOL USA, Inc.) at $15 \mathrm{kV}$. The samples were fractured in liquid nitrogen and sputtercoated with platinum (Pt). The infrared spectra of the membranes were recorded between 400 and 4,000 $\mathrm{cm}^{-1}$ on a Fourier transform infrared spectrometer (FT-IR; IRTracer-100, Shimadzu). The water contact angle, measured by a contact angle analyzer (Attension Theta, Biolin Scientific) was used to evaluate the hydrophilicity of prepared membranes by the sessile drop method of DI water $(20 \mu \mathrm{L})$. Membrane porosity $\left(P_{\mathrm{r}}\right)$ was calculated by the dry-wet weight method, as follows:

$$
P_{r}=\frac{m_{w}-m_{d}}{\rho \cdot A \cdot L}
$$


where $m_{w}$ is the wet membrane weight (g), $m_{d}$ is the dry membrane weight (g), $\rho$ is the density of distilled water $(0.998 \mathrm{~g} / \mathrm{mL}), A$ is the membrane area $\left(1 \mathrm{~cm}^{2}\right)$, and $L$ is the approximate membrane thickness $(150 \mu \mathrm{m})$.

\subsection{Ultrafiltration experiments}

Prepared membranes were characterized using a dead-end stirred-cell filtration system that was filled with DI water or a protein solution as well as applied pressure (feeding nitrogen gas). The system consisted of a filtration cell (Model 8050, Millipore Co.) with a volume capacity of 50 $\mathrm{mL}$, an inner diameter of $44.5 \mathrm{~mm}$, and an effective area (membrane) of $15.5 \mathrm{~cm}^{2}$. All the ultrafiltration experiments were carried out at a stirring speed of $300 \mathrm{rpm}$ and a temperature of $25 \pm 1{ }^{\circ} \mathrm{C}$. Each membrane was pressurized initially at $0.21 \mathrm{MPa}$ for $15 \mathrm{~min}$; then, the pressure was lowered to the operating pressure of $0.14 \mathrm{MPa}$. The initial water flux $J_{w 1}\left[\mathrm{~L} /\left(\mathrm{m}^{2} \cdot \mathrm{h}\right)\right]$ was calculated by using the following equation:

$$
J_{w 1}=\frac{V}{A \cdot \Delta t}
$$

where $V(\mathrm{~L})$ is the volume of permeated water, $A\left(\mathrm{~m}^{2}\right)$ is the membrane area, and $\Delta t$ (hour) is the permeation time. After this, the stirred cell was emptied, and refilled rapidly with protein solution $(1.0 \mathrm{mg} / \mathrm{mL}$ BSA solution). The $\mathrm{pH}$ was kept at 7.0 with a $0.1-\mathrm{M}$ phosphate buffer solution.

After ultrafiltration of the protein solution, the membranes were washed with DI water for $10 \mathrm{~min}$, and the water flux of the cleaned membranes $-J_{w 2}\left[\mathrm{~L} /\left(\mathrm{m}^{2} \cdot \mathrm{h}\right)\right]$ - were measured again. In order to evaluate the fouling-resistant ability of the membranes, the FRR was calculated using the following expression: 


$$
\mathrm{FRR}=\left(\frac{J_{w 2}}{J_{w 1}}\right) \times 100 \%
$$

\section{Results and discussion}

Fig. 4a shows the SEM image of the GO sheets, where the folded and wrinkled features of the two-dimensional thin GO sheets had a width of more than several micrometers. Figs. 4b and 4c compare the C1s narrow scans of the GO sheets and graphite from the XPS analysis. The spectra of the GO (Fig. 4b) consisted of two prominent components that arose due to the C-C bonds at $\sim 284.6 \mathrm{eV}$ and to the C-O (hydroxyl and epoxy) bonds at $\sim 286.5 \mathrm{eV}$ as well as other minor components that result from various oxygenated carbon atoms, such as $\mathrm{C}=\mathrm{O}$ and $\mathrm{O}=\mathrm{C}=\mathrm{O}$ at $\sim 288.1$ and $\sim 289.6 \mathrm{eV}$, respectively [24]. Before the oxidization process (graphite), the intensity of the C-O component was significantly lower than that of the GO (Fig. 4c). The XPS analysis indicated that the prepared GO had a hydrophilic character because of the high oxidization with hydrophilic functional groups.
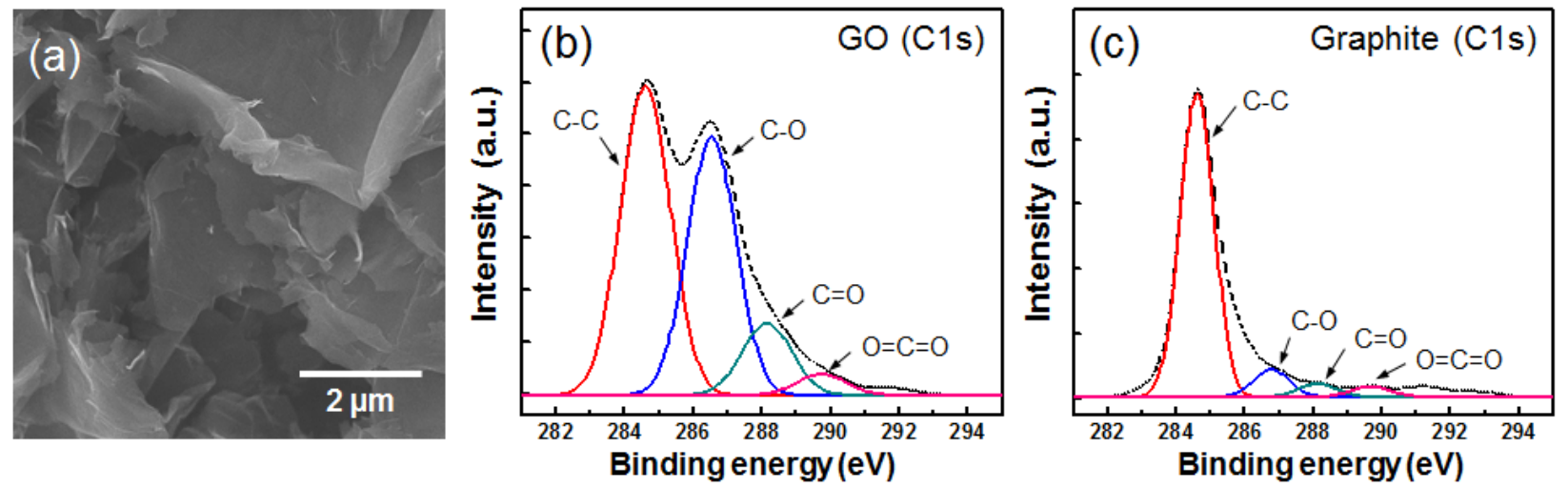

Fig. 4. The morphology of (a) prepared GO, C1s XPS spectrum of (b) GO, and (c) graphite.

Figs. 5(a-d) show the variation in the morphologies of the PSU membranes prepared with different concentrations of PVP (0, 10, and 20 wt. \%) and the PSU/GO membrane. Generally, 
asymmetric membranes are prepared by the phase inversion method. The interchange of solvent and non-solvent due to diffusion causes the casting solution to undergo a phase transition, through which the membrane is formed [1]. The pristine PSU membrane has a finger-like structure with a large number of macrovoids forms, as shown in Fig. 5(a). When increasing concentration of PVP, a finger-like structures are transformed to sponge-like structures. From these image, it is clear that the finger-like structures in the prepared membranes are suppressed by the addition of PVP. The viscosity of prepared casting solution was increased that can be attributed to increased concentration of PVP. As a result, the mutual diffusivities between components in the solution have less activated. These results are in accordance with previously reported articles $[25,26]$. Figs. 5e and $5 f$ show the surface morphology and photograph (inset) of PP10 and PSU/GO membranes, respectively. The PSU/GO membrane had the folded and wrinkled features of two-dimensional thin GO sheets in the in-plane direction, and had a slightly brown color by well-deposited GO on the PP10, as shown in Fig. $5 f$.
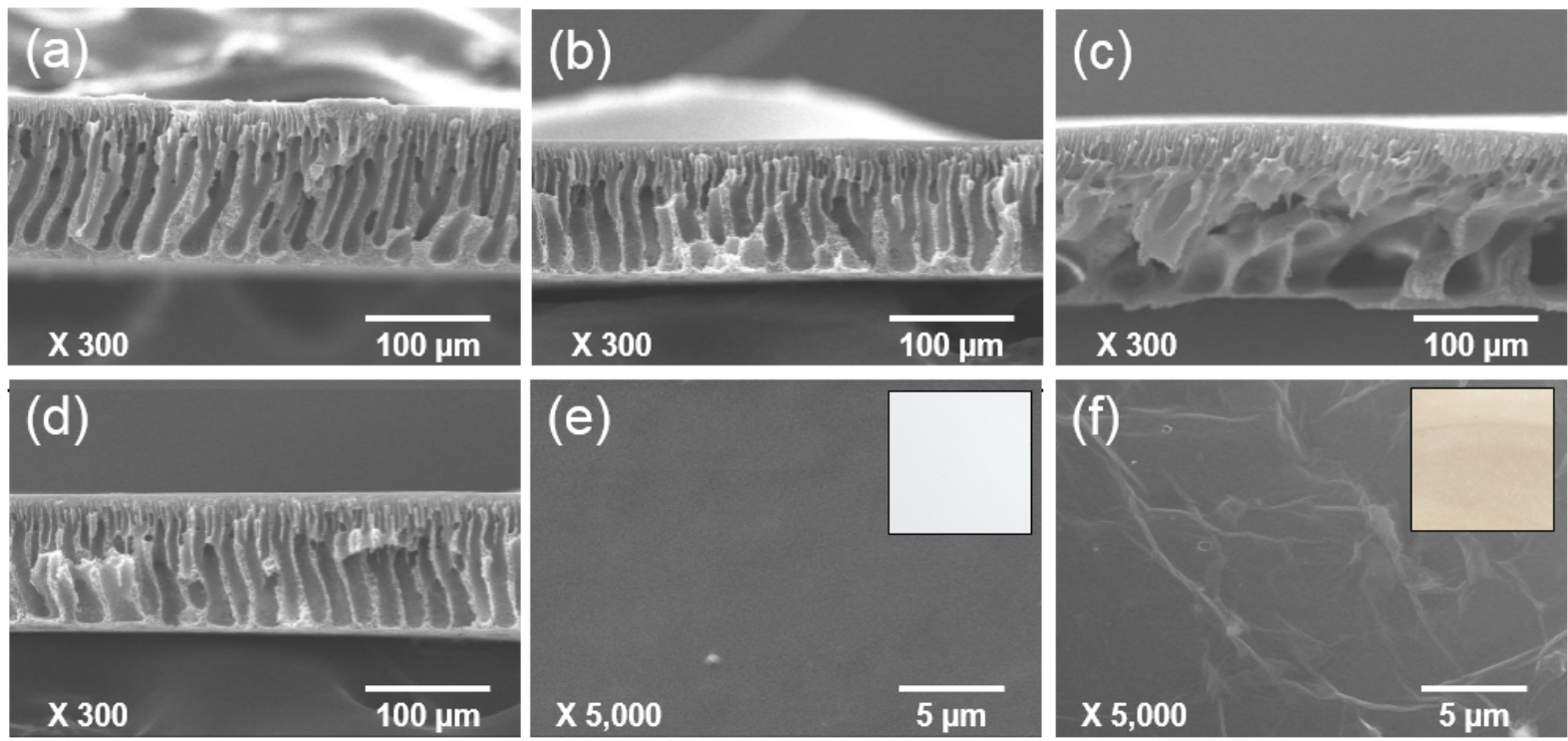

Fig. 5. The cross-section morphology of (a) PP0, (b) PP10, (c) PP20, and (d) PSU/GO. The surface morphology and photograph (inset) of (e) PP10 and (f) PSU/GO membranes. 
Fig. 6 shows the FT-IR spectra of the fabricated membranes (PP0, PP10, PP20, and PSU/GO), PVP as an additive, and GO as a hydrophilic modifier. Fig. 6a shows the FT-IR spectrum of the pristine PSU membrane (PP0). Figs. 6b and 6c show PVP-blended PSU membranes at 10 and 20 wt. \% PVP, respectively. The strong peaks at 1,150 and $1,242 \mathrm{~cm}^{-1}$ for PSU and PVP-blended membranes are associated with the stretching vibration of the $\mathrm{S}=\mathrm{O}$ and $\mathrm{C}-\mathrm{O}-\mathrm{C}$ bond of the PSU, respectively [Figs. 6(a-c)] [27]. The peak at $1,648 \mathrm{~cm}^{-1}$ is attributed to the amide carbonyl stretching of PVP, as shown in the Fig. 6e. In addition, this peak appears slightly in the spectra obtained from the PVP-blended membranes, as shown in Figs. 6b and 6c. Fig. 6f shows the spectrum of GO, which has peaks at 1,730 and 3,200-3,400 $\mathrm{cm}^{-1}$ that correspond to the C-O and -OH stretching, respectively. These findings indicate the existence of the hydrophilic functional groups in the GO. The spectrum of the PSU/GO membrane clearly shows a broad band at 3,200$3,400 \mathrm{~cm}^{-1}$ (Fig. 6d), which suggests that the hydrophilic layer formed on the surface of the membrane due to hydrophilic functional groups of the GO.

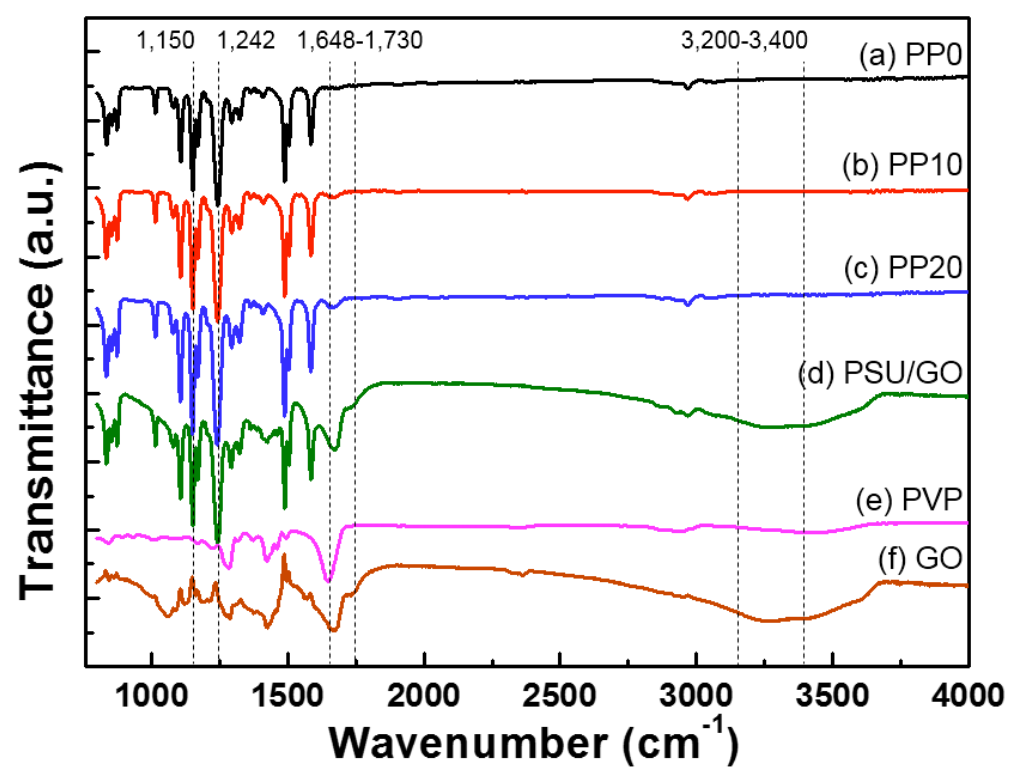


Fig. 6. FT-IR spectra of (a) PP0, (b) PP10, (c) PP20, (d) PSU/GO membranes, (e) PVP, and (f) GO.

Figs. 7(a-d) show the water contact angles of PP0, PP10, PP20, and PSU/GO membranes. Surface hydrophilicity is an important factor in determining the antifouling property of the ultrafiltration membranes. The lower contact angle represents the hydrophilic nature to wet the membrane easily, higher surface energy, and higher hydrophilicity [28]. Among all the membranes were tested, the pristine PSU membrane (Fig. 7a) shows the higher hydrophobic character with a higher contact angle of about $79.12^{\circ}$ than others. The water contact angle decreased as the amount of PVP increased, since hydrophilic functional groups were introduced to the surface of the hydrophobic PSU membrane. Fig. 7b and 7c show that the addition 10 and 20 wt. \% of PVP in the PSU solution decreased the water contact angle of the membranes to $63.15^{\circ}$ and $61.39^{\circ}$, respectively. When a layer of the hydrophilic GO was applied on the membrane surface, the water contact angle was further decreased to $42.72^{\circ}$ (Fig. 7d). Thus, a deposited layer of GO on the PSU membrane maximized the hydrophilic character of the prepared membrane by associating the hydrophilic functional group of the GO sheets, as seen from the XPS and FT-IR results. Fig 7e summarizes the results of the contact angles of the prepared membranes. 


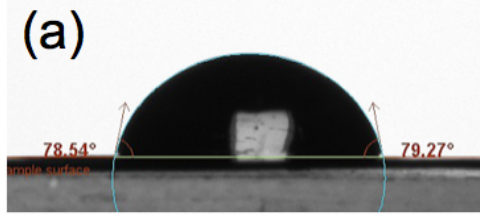

(b)
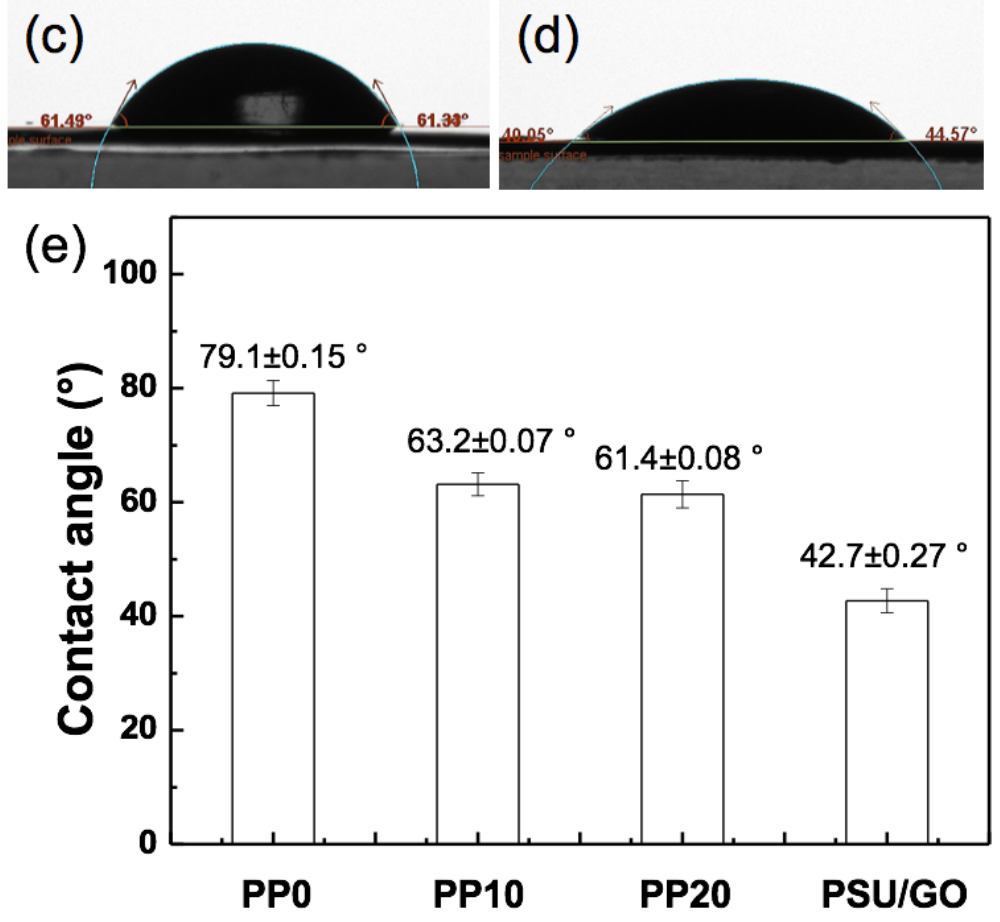

Fig. 7. Contact angles of the membranes by DI water (a) PP0, (b) PP10, (c) PP20, (d) and PSU/GO; at bottom, (e) summarized results.

Fig. 8 presents the porosity of PP0, PP10, PP20, and PSU/GO membranes. The prepared membranes (PP0, PP10, PP20, and PSU/GO) had 23.9\%, 71.0\%, 48.8\%, and 72.5\% of porosity, respectively. The hydrophilicity effect of PVP could increase the solvent and non-solvent exchange during the phase-inversion process [29]. On the other hand, when 20 wt. \% of PVP was added to the casting solution, the viscosity might be increased. This causes a slow exchange rate between the solvent and nonsolvent and resulting in a lower porosity. Chakrabarty et al. reported that the addition of an additive into the casting solution may increase the viscosity of the casting solution [30]. Also, similar observation has been reported by Yu et al. [31]. 


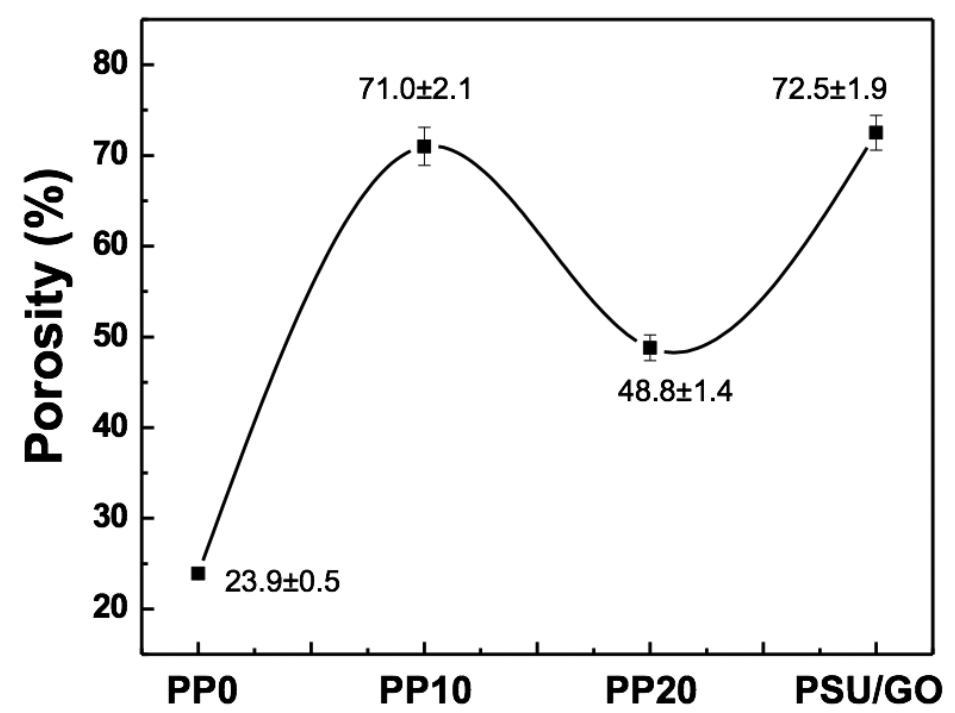

Fig. 8. Porosity of the prepared PSU membranes (PP0, 10, 20, and PSU/GO).

As shown in Figs. 9a and 9b, the initial water flux and the water FRR (after renewal of the membrane surfaces by washing with DI water) were analyzed to verify the improved antifouling performance by the addition of PVP and the hydrophilic layer of GO. Fig 9a shows the initial results of the pure water flux of the prepared membranes. The pure water flux through membranes shows the non-linear relationship with the PVP contents. The pristine PSU (PP0) had the lowest initial water flux of $25.0 \mathrm{~L} /\left(\mathrm{m}^{2} \cdot \mathrm{h}\right)$, and the prepared PP10 membrane had the highest flux of $322.2 \mathrm{~L} /\left(\mathrm{m}^{2} \cdot \mathrm{h}\right)$. However, a higher additive concentration (PP20) in the membranes led to a decrease in flux $\left[193.2 \mathrm{~L} /\left(\mathrm{m}^{2} \cdot \mathrm{h}\right)\right]$. When added high concentration of PVP in cast solution, the reduction of pure water flux would be correlated with delayed polymer coagulation on the surface region during phase inversion process. Therefore, lowering the pure water flux data with 20 wt. \% content of PVP may suggest that the balance in correlation between enhancement of thermodynamic and inhibition of rheological diffusion [26]. The layer of GO-added membrane had $309.2 \mathrm{~L} /\left(\mathrm{m}^{2} \cdot \mathrm{h}\right)$ of initial water flux, which was slightly lower than for PP10. Although PSU/GO was fabricated from PP10, one additional thin layer of GO reduced the initial water flux. 

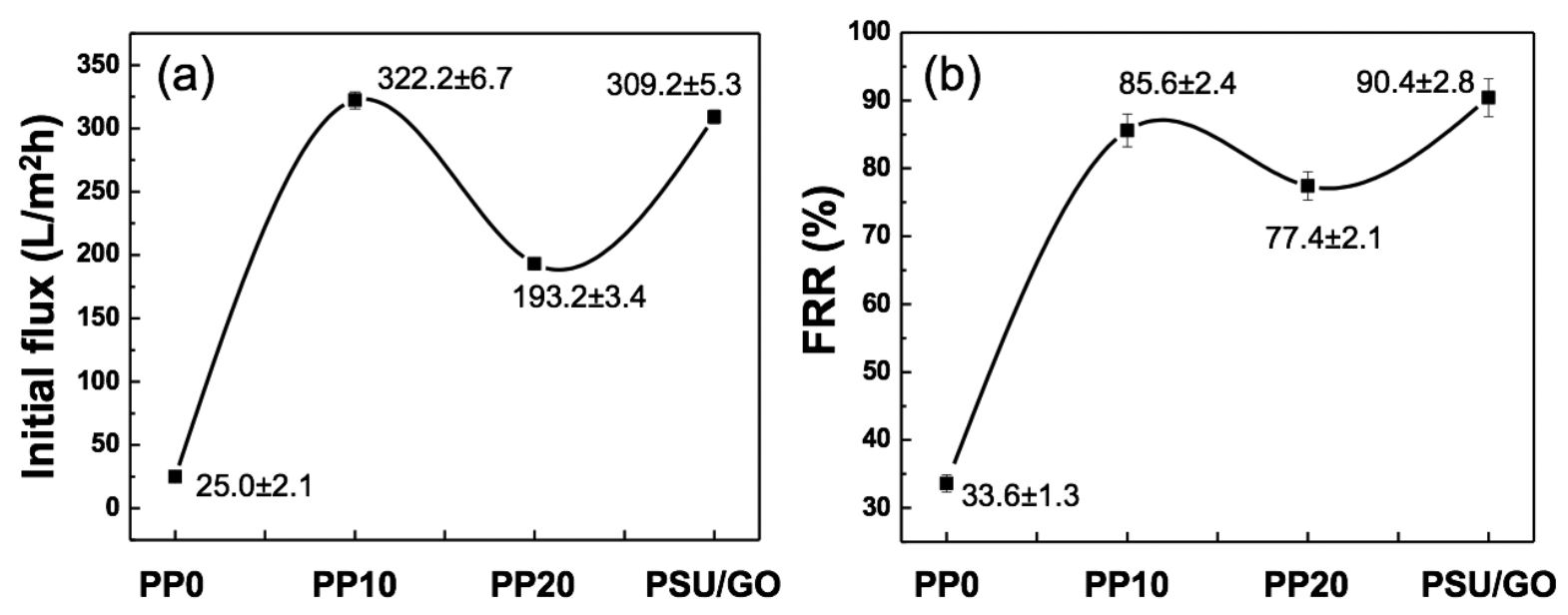

Fig. 9. Initial flux (a) and FRR results (b) of the fabricated PSU membranes (PP0, 10, 20, and PSU/GO).

The FRRs of the prepared membranes are depicted in Fig. 9b. A higher FRR indicates better antifouling performance of the membrane. The FRR for the pristine PSU membrane (33.6\%) was lower than the FRR for the membranes prepared by adding PVP or GO (more than 75.0\%). It is proposed that some protein molecules may deposit on the membrane surface or clog the membrane pore [32]. PP10, PP20, and PSU/GO had FRRs of $85.6 \%, 77.4 \%$, and $90.4 \%$, respectively. Although PSU/GO membranes showed a lower initial flux than PP10, the higher hydrophilicity of the PSU/GO membrane decreased the interactions between bio-contaminants and the membrane surface, leading to decreased contaminant adsorptions or being able to remove the fouling layers more easily [32-33]. Additionally, these experimental results indicated that the antifouling performance of the PSU/GO ultrafiltration membrane was improved by the hydrophilic nature of the GO layer. Results of the ultrafiltration membranes are summarized in Table 2.

Table 2 
Summarized results of the prepared ultrafiltration membranes.

\begin{tabular}{ccccc}
\hline Sample & $\begin{array}{c}\text { Contact Angle } \\
\left({ }^{\circ}\right)\end{array}$ & $\begin{array}{c}\text { Porosity } \\
(\%)\end{array}$ & $\begin{array}{c}\text { Pure water flux } \\
{\left[\mathrm{L} /\left(\mathrm{m}^{2} \cdot \mathrm{h}\right)\right]}\end{array}$ & $\begin{array}{c}\text { FRR } \\
(\%)\end{array}$ \\
\hline PP0 & $79.1 \pm 0.15$ & $23.9 \pm 0.5$ & $25.0 \pm 2.1$ & $33.6 \pm 1.3$ \\
PP10 & $63.2 \pm 0.07$ & $71.0 \pm 2.1$ & $322.2 \pm 6.7$ & $85.6 \pm 2.4$ \\
PP20 & $61.4 \pm 0.08$ & $48.8 \pm 1.4$ & $193.2 \pm 3.4$ & $77.4 \pm 2.1$ \\
PSU/GO & $42.7 \pm 0.27$ & $72.5 \pm 1.9$ & $309.2 \pm 5.3$ & $90.4 \pm 2.8$ \\
\hline
\end{tabular}

In addition, the lifetime and durability of PSU/GO should be an important factor to apply conventional ultrafiltration system. Therefore, the lifetime and durability would be further investigated in the future.

\section{Conclusions}

This paper described the development of novel PSU/GO ultrafiltration membranes with enhanced antifouling properties. The membranes were easily prepared by phase inversion and a vacuum-assisted filtering method. The hydrophilic layer of the GO was employed as a pre-filter to improve the hydrophilicity of the PSU membrane. The prepared membranes showed improved antifouling performance. This new route to enhancing the hydrophilic character of the surface of polymer membranes offers great potential of PSU/GO membranes as a new type of antifouling ultrafiltration membrane.

\section{Acknowledgments}

This work was partially supported by the U.S. Department of Energy/Office of Science DESC0005062. Dr. Kwang Kim would like to thank Southwest Gas Corporation for financial 
support of his Southwest Gas Professorship at UNLV for this research as well as the Korea Institute of Industrial Technology (KITECH) for international collaboration.

\section{References}

[1] E. Filloux, B. Teychene, A. Tazi-Pain, J.P. Croue, Ultrafiltration of biologically treated domestic wastewater: How membrane properties influence performance, Sep. Purif. Technol. 134 (2014) 178-186.

[2] M.K. Sinha, M.K. Purkait, Preparation and characterization of stimuli-responsive hydrophilic polysulfone membrane modified with poly (N-vinylcaprolactam-co-acrylic acid), Desalination 348 (2014) 16-25.

[3] A. Razmjou, A. Resosudarmo, R.L. Holmes, H. Li, J. Mansouri, V. Chen, The effect of modified $\mathrm{TiO}_{2}$ nanoparticles on the polyethersulfone ultrafiltration hollow fiber membranes, Desalination 287 (2012) 271-280.

[4] H.M. Hegab, Y. Wimalasiri, M. Ginic-Markovic, L. Zou, Improving the fouling resistance of brackish water membranes via surface modification with graphene oxide functionalized chitosan, Desalination 365 (2015) 99-107.

[5] I. Banerjee, R.C. Pangule and R.S. Kane, Antifouling coatings: recent developments in the design of surfaces that prevent fouling by proteins, bacteria, and marine organisms, Adv. Mater. 23 (2011) 690-718.

[6] Y.-H. Zhao, K.-H. Wee and R. Bai, Highly hydrophilic and low-protein-fouling polypropylene membrane prepared by surface modification with sulfobetaine-based zwitterionic polymer through a combined surface polymerization method, J. Membr. Sci. 362 (2010) 326-333. 
[7] S.P. Roux, E.P. Jacobs, A.J.V. Reenen, C. Morkel and M. Meincken, Hydrophilisation of polysulphone ultrafiltration membranes by incorporation of branched PEO-blockPSU copolymers, J. Membr. Sci. 276 (2006) 8-15.

[8] R. Lv, J. Zhou, Q. Du, H. Wang and W. Zhong, Preparation and characterization of EVOH/PVP membranes via thermally induced phase separation, J. Membr. Sci. 281 (2006) 700-706.

[9] J.-F. Li, Z.-L. Xu, H. Yang, L.-Y. Yu and M. Liu, Effect of $\mathrm{TiO}_{2}$ nanoparticles on the surface morphology and performance of microporous PES membrane, Appl. Surf. Sci. 255 (2009) 4725-4732.

[10] G. Liu, L. Zhang, S. Mao, S. Rohani, C. Ching, J. Lu, Zwitterionic chitosan-silica-PVA hybrid ultrafiltration membranes for protein separation, Sep. Purif. Technol. 152 (2015) 55-63.

[11] A. Ananth, G. Arthanareeswaran and H. Wang, The influence of tetraethylorthosilicate and polyethyleneimine on the performance of polyethersulfone membranes, Desalination 287 (2012) 61-70.

[12] J. Garcia-Ivars, M.-I. Alcaina-Miranda, M.-I. Iborra-Clar, J.-A. Mendoza-Roca, L. Pastor-Alcañiz, Enhancement in hydrophilicity of different polymer phase-inversion ultrafiltration membranes by introducing PEG/ $/ \mathrm{Al}_{2} \mathrm{O}_{3}$ nanoparticles, Sep. Purif. Technol. 128 (2014) 45-57.

[13] G. Arthanareeswaran and P. Thanikaivelan, Fabrication of cellulose acetate-zirconia hybrid membranes for ultrafiltration applications: Performance, structure and fouling analysis, Sep. Purif. Technol. 74 (2010) 230-235.

[14] K.R. Kull, M.L. Steen and E.R. Fisher, Surface modification with nitrogen-containing 
plasmas to produce hydrophilic, low-fouling membranes, J. Membr. Sci. 246 (2005) 203-215.

[15] M.J. Han, G.N.B. Baroña and B. Jung, Effect of surface charge on hydrophilically modified poly(vinylidene fluoride) membrane for microfiltration, Desalination 270 (2011) 76-83.

[16] X. Wang, C. Chen, H. Liu and J. Ma, Preparation and characterization of PAA/PVDF membrane-immobilized Pd/Fe nanoparticles for dechlorination of trichloroacetic acid, Water Res. 42 (2008) 4656-4664.

[17] N. Meng, Z. Wang, Z.-X. Low, Y. Zhang, H. Wang, X. Zhang, Impact of trace graphene oxide in coagulation bath on morphology and performance of polysulfone ultrafiltration membrane, Sep. Purif. Technol. 147 (2015) 364-371.

[18] S. Majeed, D. Fierro, K. Buhr, J. Wind, B. Du, A. Boschetti-de-Fierro and V. Abetz, Multi-walled carbon nanotubes (MWCNTs) mixed polyacrylonitrile (PAN) ultrafiltration membranes, J. Membr. Sci. 403-404 (2012) 101-109.

[19] E. Celik, L. Liu and H. Choi, Protein fouling behavior of carbon nanotube/polyethersulfone composite membranes during water filtration, Water Res. 45 (2011) 5287-5294.

[20] T. Ghosh, C. Biswas, J. Oh, G. Arabale, T. Hwang, N.D. Luong, M. Jin, Y.H. Lee and J.D. Nam, Solution-processed graphite membrane from reassembled graphene oxide, Chem. Mater. 24 (2012) 594-599.

[21] J.S. Oh, T. Hwang, G.Y. Nam, J.P. Hong, A.H. Bae, S.I. Son, G.H. Lee, H.K. Sung, H.R. Choi, J.C. Koo and J.D. Nam, Chemically-modified graphene sheets as an active layer for eco-friendly metal electroplating on plastic substrates, Thin Solid Films 521 (2012) 
270-274.

[22] F. Jin, W. Lv, C. Zhang, Z. Li, R. Su, W. Qi, Q.-H. Yang and Z. He, High-performance ultrafiltration membranes based on polyethersulfone-graphene oxide composites, RSC Adv. 3 (2013) 21394-21397.

[23] N.V. Medhekar, A. Ramasubramaniam, R.S. Ruoff, V.B. Shenoy, Hydrogen bond networks in graphene oxide composite paper: structure and mechanical properties, ACS Nano 4 (2010) 2300-2306.

[24] H.-J. Shin, K.K. Kim, A. Benayad, S.-M. Yoon, H.K. Park, I.-S. Jung, M.H. Jin, H.-K. Jeong, J.M. Kim, J.-Y. Choi and Y.H. Lee, Efficient reduction of graphite oxide by sodium borohydride and its effect on electrical conductance, Adv. Funct. Mater. 19 (2009) 1987-1992.

[25] S.H. Yoo, J.H. Kim, J.Y. Jho, J. Won, Y.S. Kang, Influence of the addition of PVP on the morphology of asymmetric polyimide phase inversion membranes: effect of PVP molecular weight, J. Membr. Sci. 236 (2004) 203-207.

[26] M.-J. Han, S.-T. Nam, Thermodynamic and rheological variation in polysulfone solution by PVP and its effect in the preparation of phase inversion membrane, J. Membr. Sci. 202 (2002) 55-61.

[27] R. Kumar, A.M. Isloor, A.F. Ismail, S.A. Rashid and T. Matsuura, Polysulfone-chitosan blend ultrafiltration membranes: preparation, characterization, permeation and antifouling properties, RSC Adv. 3 (2013) 7855-7861.

[28] Y. Liu, X. Yue, S. Zhang, J. Ren, L. Yang, Q. Wang and G. Wang, Synthesis of sulfonated polyphenylsulfone as candidates for antifouling ultrafiltration membrane, Sep. Purif. Technol. 98 (2012) 298-307. 
[29] Y. Yang, H. Zhang, P. Wang, Q. Zheng and J. Li, The influence of nano-sized $\mathrm{TiO}_{2}$ fillers on the morphologies and properties of PSF UF membrane, J. Membr. Sci. 288 (2007) 231-238.

[30] B. Chakrabarty, A.K. Ghoshal, M.K. Purkait, Preparation, characterization and performance studies of polysulfone membranes using PVP as an additive, J. Membr. Sci. 315 (2008) 36-47.

[31] L. Yu, Y. Zhang, B. Zhang, J. Liu, H. Zhang, C. Song, Preparation and characterization of HPEI-GO/PES ultrafiltration membrane with antifouling and antibacterial properties, J. Membr. Sci. 447 (2013) 452-462.

[32] K.A. Mahmoud, B. Mansoor, A. Mansour, M. Khraisheh, Functional graphene nanosheets: The next generation membranes for water desalination, Desalination 356 (2015) 208-225.

[33] J. Lee, H.-R. Chae, Y.J. Won, K. Lee, C.-H. Lee, H.H. Lee, I.-C. Kim, J.-m. Lee, Graphene oxide nanoplatelets composite membrane with hydrophilic and antifouling properties for wastewater treatment, J. Membr. Sci. 448 (2013) 223-230. 